\title{
Non-axisymmetric and/or non-elementary response of anisotropic tuff in axisymmetric, elementary triaxial test
}

\author{
Yota Togashi ${ }^{1, *}$, Mamoru Kikumoto ${ }^{2}$, Kazuo Tani $^{3}$, Koichi Hosoda $^{4}$, and Koji Ogawa ${ }^{4}$ \\ ${ }^{1}$ Saitama university, 255 Shimo-Okubo Sakura Saitama, Japan \\ ${ }^{2}$ Yokohama National University, 79-5 Tokiwadai Hodogaya Yokohama, Japan \\ ${ }^{3}$ Tokyo University of Marine Science and Technology, 4-5-7 Konan Minato Tokyo, Japan \\ ${ }^{4}$ OYO corporation, 1-66-2 Miyaharacho Kita Saitama, Japan
}

\begin{abstract}
Specimens of triaxial tests ordinarily response as element in the axisymmetric condition, and it is theoretically correct assumption. However, for anisotropic rocks, the axisymmetric condition is not always satisfied because incremental relationships between stresses and strains are not coaxial. In addition, such rock specimen often shows non-uniform deformation behaviors due to end frictions. As the reason, the influence of end restraints on deformation behaviors of anisotropic rocks during triaxial compression is much greater than that of isotropic one. In this study, the variations in the stress-strain relationships of anisotropic tuff due to the condition of end restraints are investigated. The results of four consolidated drained triaxial tests with measuring the strain tensor demonstrated that the axial and volumetric strains were not influenced by whether sliding mechanism and Teflon sheets are installed at the end of specimens or not; meanwhile, the shear components of the strain tensor and the inclinations of principal strain orientations are widely varied owing to the end restraints.
\end{abstract}

\section{Introduction}

Deformation properties of sedimentary rocks become anisotropic due to sedimentary structures. For example, the Young's moduli of the tuff at neogene period vary by a factor of 2.7 due to the bedding orientation[1]; that of the mudstone at same period vary by a factor of 2 due to sampling orientations[2]. It is significantly important to grasp such anisotropy for the design and constructions of the rock structures (e.g. tunnel, foundation and so on).

Mechanical properties of rocks can be ordinarily evaluated by triaxial or uniaxial test. It is much important for evaluating the mechanical properties accurately by using the tests to install frictionless condition on the boundary between the specimen ends and the cap or pedestal. The influence of end restraints on the specimen and that of the ratio of the specimen height to diameter, $h / d$, has been investigated for long years. Bishop and Green [3] indicated that friction angle of soil $\phi$ is overestimated in the case of $h / d<2.0$ owing to end restraints. Based on the study, the cylindrical specimen of $h / d \geq 2.0$ is recommended for the triaxial soil test by Japanese Geotechnical Standards[10]. Furthermore, the stress-strain relationships of rock specimens are often influenced by local deformations on the ends of the specimen. This may occur when the ends of stiff specimens (like rocks) are not completely parallel, and this is called bedding error [5]. To prevent bedding error, the specimen of $1.8 \leq h / d \leq 2.5$ is recommended for the triaxial test using rock specimens by

*e-mail: togashi@mail.saitama-u.ac.jp
Japanese Geotechnical Standard [10]. In addition, some methods are developed to measure the local deformation of the middle of the specimen for neglecting the influence of local deformations at the ends of a specimen (e.g. [6]).

Incidentally, the anisotropic rock specimen often shows non-axisymmetric deformations as shown in Fig.1 because the stress-strain relationships are not coaxial [7][8]. In the frictionless condition in Fig.1(a), the principal orientation of strains are rotated and the shear deformation can be uniformly obtained. In the frictional condition in Fig.1(b), the end planes of the specimen are completely fixed to the lateral orientation. The principal orientations of strains are agreed with the loading axis, and those of stresses are inclined. In addition, the principal orientations of stresses at the center of the specimen are close to the loading axis owing to no lateral restraints. As a result, the deformation of the specimen become complicated and the correct results cannot be evaluated. As the comparison of these condition, the specimen in frictionless condition (Fig.1(a)) can be responded as element. Thus, we stated the importance of decreasing the end friction and a new cap was developed for anisotropic rocks [2].

In this study, four triaxial specimens of the cylindrical shape of $h / d=2.0$ are made by the rhyolitic welded tuff including clear bedding planes. Then, the series of consolidated-drained triaxial tests in four conditions of end restraints are conducted. Whether sliding mechanism and Teflon sheets are installed or not is investigated to evaluate the influence of end restraints. Firstly, the variations in strain responses are compared in each case by 




Fig. 1. Non axisymmetric deformation of triaxial specimen

strain tensor measurement using three rosetta gages. Then, that of the rotations of principal strain orientations due to end frictions are also compared.

\section{Method}

Fig. 2 shows the structure of a new cap with sliding mechanism [7]. Circular shape ball bearings are installed on the boundary between top and bottom platen. Then, two-ply Teflon sheets (static friction angle $=0.04$, [9]) with silicon grease are installed on the top and bottom of specimen to decrease the friction on the boundary between the cap and the specimen, as shown in Fig.1.



Fig. 2. A new cap with sliding mechanism

In this study, the variations of stress-strain behaviors are discussed by four test specimens (Table.1) in different boundary conditions of whether the sliding mechanism and Teflon sheets are installed or not.

Table 1. Test cases

\begin{tabular}{cccc}
\hline Case & $\begin{array}{c}\text { Wet density } \\
\rho_{t}\left(\mathrm{~g} / \mathrm{cm}^{3}\right)\end{array}$ & $\begin{array}{c}\text { Sliding } \\
\text { mechanism }\end{array}$ & $\begin{array}{c}\text { Teflon } \\
\text { sheet }\end{array}$ \\
\hline 1 & 1.72 & $\checkmark$ & $\checkmark$ \\
2 & 1.71 & $\checkmark$ & $\times$ \\
3 & 1.71 & $\times$ & $\checkmark$ \\
4 & 1.72 & $\times$ & $\times$ \\
\hline
\end{tabular}

The sample used for the test is rhyolitic welded tuff at Neogene period. The tuff were sampled at $100 \mathrm{~m}$ underground of Utsunomiya city, Japan. The cylindrical specimens (diameter $d=5 \mathrm{~cm}$ and height $h=10 \mathrm{~cm}$ ) were cored from $30 \mathrm{~cm}$ square block. The bedding plane is inclined by $30^{\circ}$ from the end of the specimen as Fig. 3 .
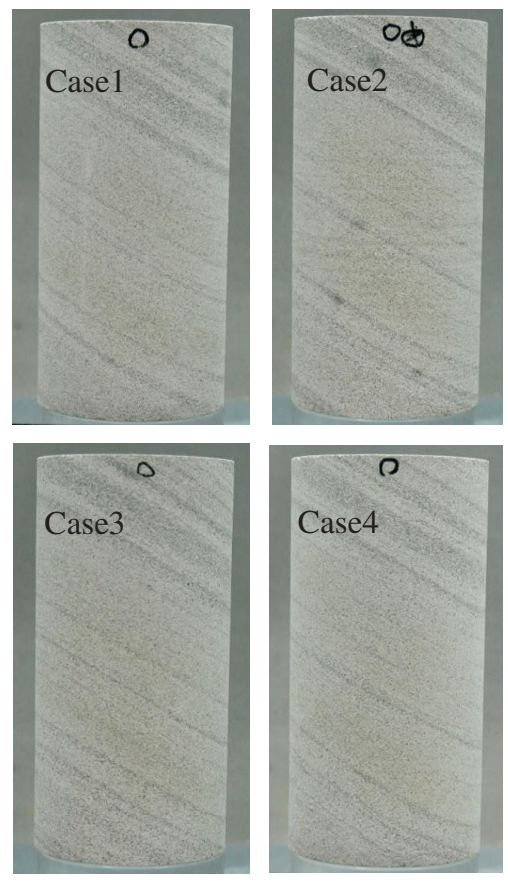

Fig. 3. Tuff specimens

The specimens were saturated by 48 hours water immersion before the test [10]. The variations in wet density, $\rho_{t}$, are very few as $\rho_{t}=1.71-1.72 \mathrm{~g} / \mathrm{cm}^{3}$. The ends of specimens were shaped by smaller parallelisms than $0.05 \mathrm{~mm}$ [11]. The specimens were isotropically consolidated by mean effective confining stress $\sigma_{c}^{\prime}=1.0 \mathrm{MPa}$ (cell pressure $=1.2 \mathrm{MPa}$, back pressure $=0.2 \mathrm{MPa}$ ), and after strains become stable, the specimens were axial compressed under drained condition by $0.03 \% / \mathrm{min}$ as the precious study [1]. The axial loading and unloading are repeated at three stress states $\Delta \sigma_{a}=1.0 \mathrm{MPa}, 3.0 \mathrm{MPa}$ and 6.0MPa, respectively, as shown in Fig.4.

The deformation behaviors of specimens are measured in detail by the method as shown in Fig.5. The small strain tensor in the $(X, Y, Z)$ coordinate is measured by three rosette gauges attached on the middle of the specimens by $120^{\circ}$ interval. As the dip angle of the bedding plane is described by the rotation of $X$ axis in $(X, Y, Z)$ coordinate, the relationship between the six components of small strain tensor $\boldsymbol{\varepsilon}$ and normal strains $\mathbf{x}$ measured by three rosette gauges is given by following equation.

$$
\boldsymbol{\varepsilon}=\left(\mathbf{E}^{\mathrm{T}} \mathbf{E}\right)^{-1} \mathbf{E}^{\mathrm{T}} \mathbf{x}
$$

$\mathbf{E}$ is $9 \times 6$ matrix determined by unit normal vector of 9 strain gauges as given in detail in [2]. Furthermore, the components of strain tensor $\boldsymbol{\varepsilon}$ are determined from $\mathbf{x}$ using least square method (Eq.(1)). 



Fig. 4. Stress pass; the relationships of axial stress with axial strain, that of time with axial strain

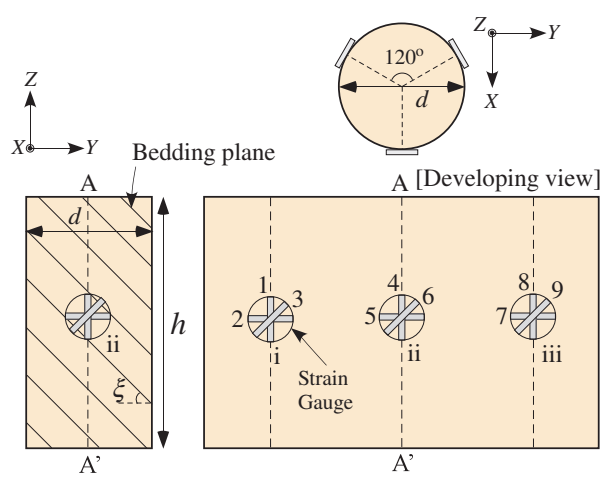

Fig. 5. Setting of strain gauges

\section{Test results and discussion}

Table 2 shows the values of small strain tensor in a stable state after isotropic consolidation. Normal and shear components of the strain tensor are positive to compressive orientations and to anti-clockwise orientation, respectively.

Table 2. Strain tensor during isotropic consolidation

\begin{tabular}{ccccccccc}
\hline Case & $t$ & $\sigma_{c}^{\prime}$ & \multicolumn{5}{c}{$\varepsilon_{i j}(\%)(i, j=X, Y, Z)$} \\
\cline { 5 - 9 } & $(\mathrm{min})$ & $(\mathrm{MPa})$ & $X X$ & $Y Y$ & $Z Z$ & $X Y$ & $Z Y$ & $Z X$ \\
\hline 1 & 61 & 1.00 & 0.040 & 0.066 & 0.041 & -0.014 & 0.060 & -0.028 \\
2 & 76 & 0.99 & 0.038 & 0.071 & 0.049 & -0.008 & 0.015 & 0.006 \\
3 & 61 & 0.99 & 0.030 & 0.048 & 0.051 & -0.006 & 0.018 & 0.005 \\
4 & 75 & 1.00 & 0.034 & 0.052 & 0.056 & -0.005 & 0.018 & 0.008 \\
\hline
\end{tabular}

Anisotropic deformation behaviors are observed because normal strains get different values in each case. If the material is isotropic, normal strains show the same value and shear strains theoretically show the values of zero. For comparison of the variations, similar values are obtained in each component of normal strains as strains of $X X, Y Y$, and $Z Z$ are given by $0.03 \%-0.038 \%, 0.048 \%$ $0.071 \%$, and $0.041 \%-0.056 \%$, respectively. On the other hand, the variations in shear strains are relatively large, and the shear strains of case 1 are greater than other cases. This is because case 1 is most frictionless condition and the shear strains in other cases are decreased owing to end frictions. Table 3 shows principal strains in a stable state after isotropic consolidation.

Table 3. Principal strains after isotropic consolidation

\begin{tabular}{cccccc}
\hline Case & $t$ & $\sigma_{c}^{\prime}$ & \multicolumn{3}{c}{$\varepsilon_{i}(\%)(i=1,2,3)$} \\
\cline { 4 - 6 } & $(\min )$ & $(\mathrm{MPa})$ & 1 & 2 & 3 \\
\hline 1 & 61 & 1.00 & 0.125 & 0.034 & -0.012 \\
2 & 76 & 0.99 & 0.080 & 0.048 & 0.030 \\
3 & 61 & 0.99 & 0.068 & 0.038 & 0.023 \\
4 & 75 & 1.00 & 0.072 & 0.044 & 0.026 \\
\hline
\end{tabular}

The values are obtained by diagonalizing the strain tensor in Table 2. The values of maximum, intermediate, and minimum principal stains are varied in each case due to the anisotropic nature.

Figure 6 shows the principal strain orientations in the stable state after isotropic consolidation by Ulf net projection of lower hemisphear. The non co-axiality of stresses and strains is certainly observed because the inclinations of the principal strains cannot be observed in isotropic materials. The orientations theoretically coincide with dominant orientations of anisotropy during isotropic consolidation [12], and the rotation of the maximum principal strain orientation in accordance with the bedding orientation is confirmed by previous study [1]. Then, the bedding orientations are shown in green line, and the normal plane of the maximum principal strain orientation shows red line. The data of case1, case3, case 4 are reasonable results because the angle of inclination of maximum principal strain is 1.3-1.5 greater than the dip angle of the bedding plane as in the previous study [1]. The non co-axiality of tuff is well observed especially in case 1 and case 2 , because of installing the sliding mechanism.

Figure 7 compares normal strain increments during axial compression after 3 cycle of loading and unloading in each case. The three cycles of loading and unloadings are omitted for visibility. Axial strain increment $\Delta \varepsilon_{z}$ is dominant to the compressive orientation, and lateral strains of $\Delta \varepsilon_{x}$ and $\Delta \varepsilon_{y}$ are produced to the tensile orientation. The variations in each normal strain component are very few. The peak of volumetric strain $\varepsilon_{v}$ is $0.2 \%$ in each case, and volume of specimens are expanded after that.

Figure 8 also compares the shear strain increments during axial compression after 3 cycles of the loading and unloading in each case. On the contrary of the normal strain increments, the shear strain increments clearly show different tendencies in each case, even if the val- 



Fig. 6. Principal strain orientations during isotropic consolidation

Fig. 7. Variations in normal strain increments during axial compression; a, b, c, and d are $\Delta \varepsilon_{X X}, \Delta \varepsilon_{Y Y}, \Delta \varepsilon_{Z Z}$ and $\Delta \varepsilon_{v}$, respectively 

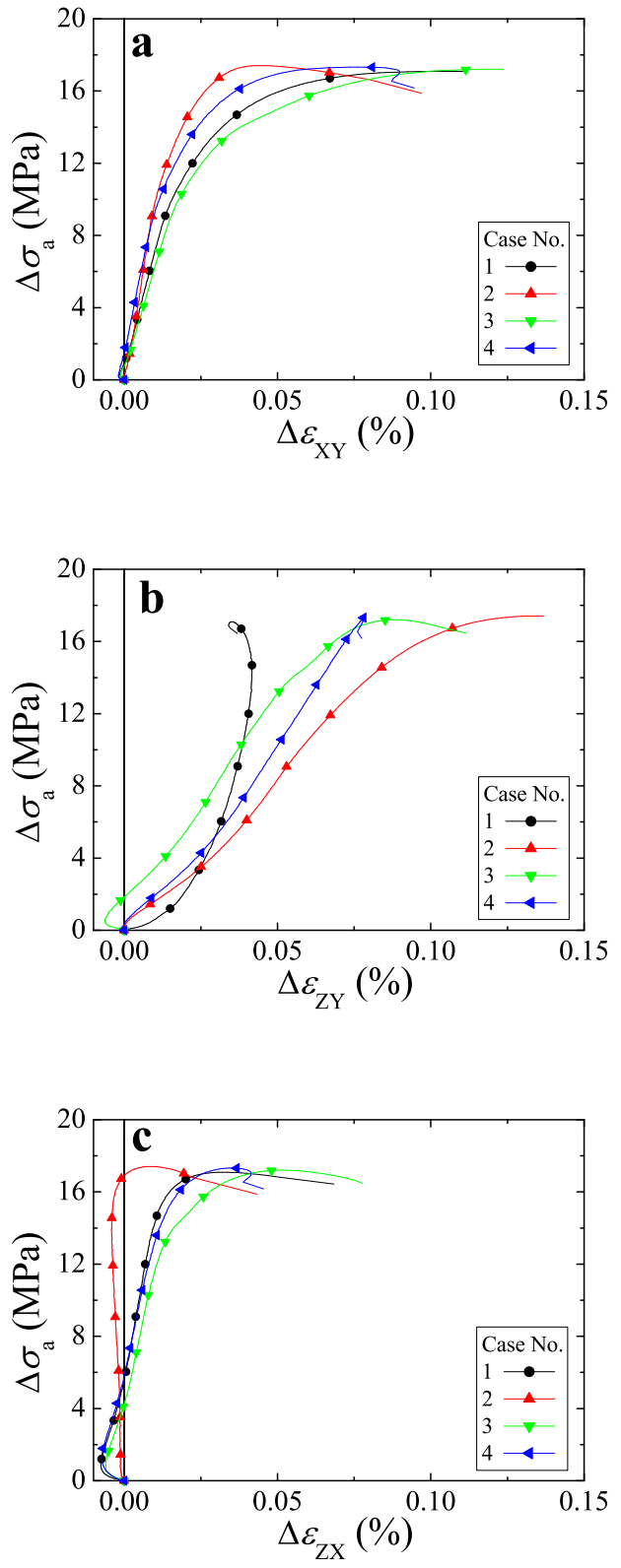

Fig. 8. Variations in shear strain increments during axial compression; $\mathrm{a}, \mathrm{b}$ and $\mathrm{c}$ are $\Delta \varepsilon_{X Y}, \Delta \varepsilon_{Z Y}$, and $\Delta \varepsilon_{Z X}$, respectively

ues are smaller than that of normal components. The shear strain component to strike orientation, $\Delta \varepsilon_{Z Y}$, especially shows quite different values, and case 1 is dominant in $\Delta \varepsilon_{Z Y} \leq 0.25 \%$ due to end friction. Although the different tendency is also obtained in $\Delta \varepsilon_{Z X}$ of case2, the values are smaller than that of $\Delta \varepsilon_{Z Y}$.

Figure 9 compares the three principal strain increments calculated by the strain tensor as shown in Fig.7 and Fig8 in each case. The variations in the principal strain increments are also quite small, and this is similar as normal strain increments. However, the shear strains are much varied as shown in Fig.8, and the orientations of principal strains should be rotated. Figure 10 shows orientations of principal strains during axial compression.
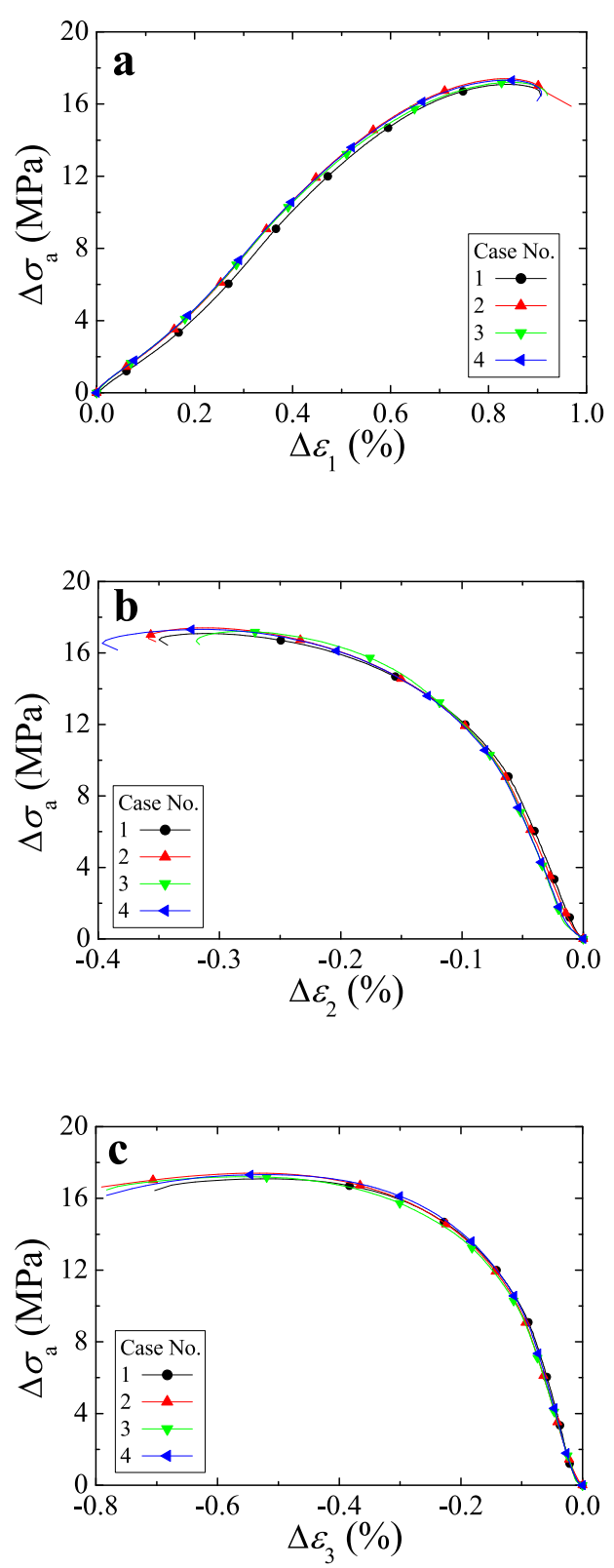

Fig. 9. Variations in principal strain increments during axial compression; $\mathrm{a}, \mathrm{b}$ and $\mathrm{c}$ are $\Delta \varepsilon_{1}, \Delta \varepsilon_{2}$ and $\Delta \varepsilon_{3}$, respectively

The continuous rotation of the principal orientations are shown by line, and three different stress states are shown by different shapes of the plots. The values of orientations are much varied due to end friction. The line of $\Delta \varepsilon_{1}$ are rotated near loading orientation $Z$, and the values of the cases possessing sliding mechanism are greater than that of the other cases. The lines of $\Delta \varepsilon_{2}$ and $\Delta \varepsilon_{3}$ are discontinuous in the some parts, because $\Delta \varepsilon_{2}$ is interchanged with $\Delta \varepsilon_{3}$, and the unit vector of $\Delta \varepsilon_{2}$ and $\Delta \varepsilon_{3}$ are also moved to upper hemisphere $(Z>0)$. As the triaxial test results explained above, although the variation in normal strains were small, that in shear strains are still large due to end friction. Furthermore, the rotations of the prin- 

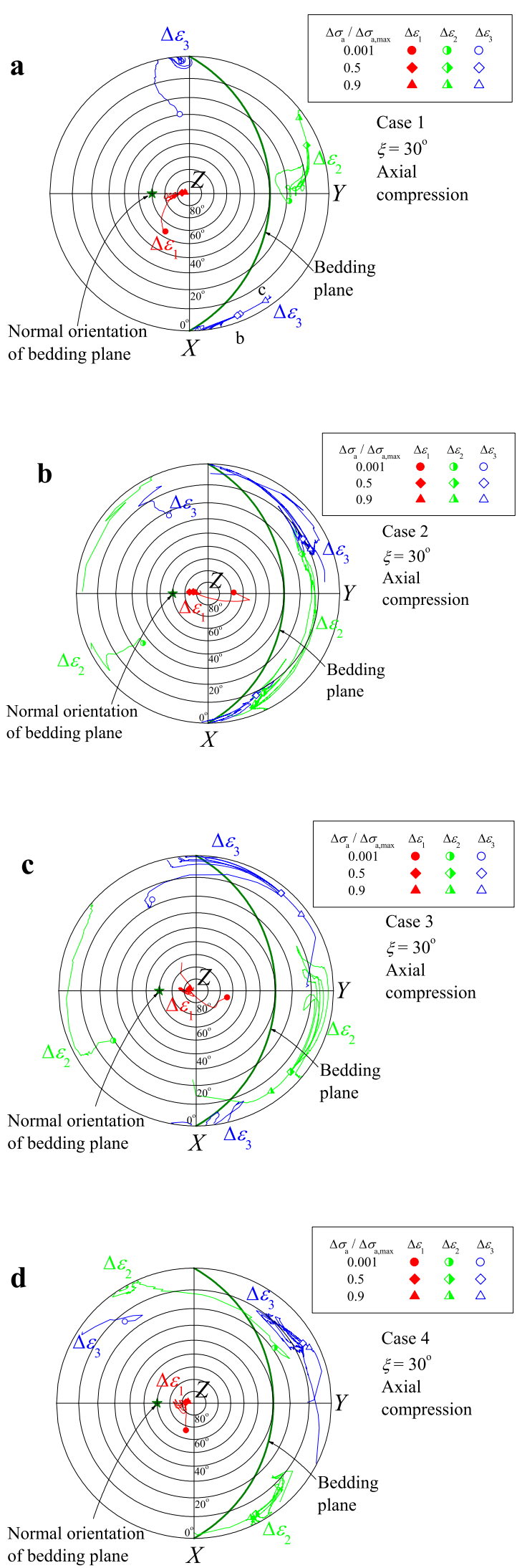

Fig. 10. Orientations of principal strain increments during axial compression cipal strain orientations are also varied owing to the end frictions.

\section{Conclusion}

The influence of end frictions on anisotropic rocks during a triaxial compression test should be quite large because the stress-strain relationships of anisotropic materials are non-coaxial, and then, the deformations of the specimen can be non-axisymmetric. In this study, to evaluate the influence on the stress-strain properties, four cases of consolidated-drained triaxial tests of rhyolitic welded tuff including clear bedding plane by using the standard size of the cylindrical specimen $(h / d=2.0)$ were conducted under both the frictionless and frictional conditions. The results of whether Teflon sheets and the sliding mechanism are installed or not on the ends of specimen, demonstrated the following conclusions. The normal strain components during both isotropic consolidation and triaxial compression get similar values in the whole cases. However, the clear difference in shear strain components are observed due to end frictions. The values of shear strains decrease in the frictional conditions, and then the difference of inclinations of the principal strains are appeared in accordance with the friction.

\section{References}

[1] Y. Togashi, M. Kikumoto, K. Tani, K. Hosoda, K. Ogawa, Int. J. Rock Mech. Min. Sci. 108, 23-36 (2018)

[2] Y. Togashi, M. Kikumoto, K. Tani, K. Hosoda and K. Ogawa, J. Soc. Mater. Sci. Jp. 67 3, 338-345 (2018)

[3] AW. Bishop, GE Green, Géotechnique 15 3, 243-266 (1965)

[4] Japanese Geotechnical Society, JGS0524-2009

[5] F. Molenkamp, F. Tatsuoka, J. Geotech. Eng. 109 5, 766-771 (1983)

[6] P. Bésuelle, J. Desrues, S. Raynaud, Int. J. Rock Mech. Min. Sci. 37 8, 1223-1237 (2000)

[7] Y. Togashi, M. Kikumoto, K. Tani, Rock Mech. Rock Eng. 50 1, 1-15 (2017)

[8] Y. Togashi, M. Kikumoto, K. Tani, J. Petroleum Sci. Eng. 169, 184-199 (2018)

[9] F. Tasuoka, F. Molenkamp, T. Torii, T. Hino, Soils Found. 24 1, 113-128 (1984)

[10] JGS2534-2009, Method for consolidated-drained triaxial compression test on rocks (2009)

[11] B JIS 0621, 0021-1974 (1974)

[12] D. Allirot, JP. Boehler, A. Sawczuk, Int. J. Rock Mech. Min. Sci. 14 2, 77-83 (1977) 